Preprint-KUL-TF-91/17

US-FT/5-91

April 1991

\title{
INTEGRABILITY AND BIHAMILTONIAN STRUCTURE OF THE EVEN ORDER SKdV HIERARCHIES
}

José M. Figueroa-O'FArrill ${ }^{1 \natural}, J_{\text {Avier }}$ Mas $^{2 b}$, and Eduardo Ramos ${ }^{1 \sharp}$

${ }^{1}$ Instituut voor Theoretische Fysica, Universiteit Leuven, Celestijnenlaan 200 D, B-3001 Heverlee, BELGIUM

${ }^{2}$ Departamento de Física de Partículas Elementales, Facultad de Física, Universidad de Santiago, Santiago de Compostela 15706, SPAIN

\begin{abstract}
We study reductions of the even order SKP hierarchy. We prove that these systems are integrable and bihamiltonian. We derive an infinite set of independent polynomial conservation laws, prove their nontriviality, and derive Lenard relations between them. A further reduction of the simplest such hierarchy is identified with the supersymmetric KdV hierarchy of Manin and Radul. We prove that it inherits all the bihamiltonian and integrability properties from the unreduced hierarchy.
\end{abstract}

Ł e-mail: fgbda11@blekul11.BITNET. Address after October 1991: Physikalisches Institut der Universität Bonn, Germany.

b e-mail: jamas@euscvx.decnet.cern.ch.

\# e-mail: fgbda06@blekul11.BITNET. 


\section{$\S 1$ INTRODUCTION}

In this paper we study a class of supersymmetric integrable systems which are obtained as reductions of a supersymmetric analog of the Kadomtsev-Petviashvili (KP) hierarchy.

The KP hierarchy ${ }^{[\mathbf{1}]}$ can be defined as the Lax-type evolution equations

$$
\frac{\partial L}{\partial t_{n}}=\left[L_{+}^{n}, L\right]=\left[L, L_{-}^{n}\right]
$$

where $L=\partial+\sum_{i \geq 0} a_{i} \partial^{-i}$ is a (formal) pseudodifferential operator and + just projects to the differential part. This hierarchy is formally integrable in the sense that it contains an infinite number of nontrivial conservation laws and an infinite number of commuting flows. Moreover a lot of completely integrable bihamiltonian systems can be obtained from it by reduction. Namely, if we impose $L_{-}^{k}=0$, we obtain the $k^{\text {th }}$ order generalized KdV hierarchy. A further reduction $\left(a_{0}=0\right)$ yields, for $k=2$ and $k=3$, the Korteweg-de Vries and Boussinesq hierarchies respectively. The generalized KdV hierarchies inherit both its conservation laws and its commuting flows from the KP hierarchy, but moreover, these are now connected by the (bi)hamiltonian structure. For details on these hierarchies we refer the reader to the review paper of Drinfel'd and Sokolov ${ }^{[\mathbf{2}]}$ and to the forthcoming book of Dickey ${ }^{[3]}$.

In [4] Manin and Radul introduced an integrable supersymmetric hierarchy (SKP) having the KP hierarchy as a natural reduction. The evolution equations are defined analogously to those of the KP hierarchy, but with the basic operator being a superpseudodifferential operator (S $\Psi D O) \Lambda=D+\sum_{i \geq 0} A_{i} D^{-i}$ with $D$ (resp. $A_{i}$ ) the supercovariant derivative (resp. superfields) on a (1|1) superspace (see later). This hierarchy is also integrable in the same sense that the KP hierarchy is. Imposing the constraint $\Lambda_{-}^{k}=0$ one can also get integrable hamiltonian hierarchies, but unlike in the KP case one does not obtain the most general $k^{\text {th }}$ order Lax hierarchy this way unless $k$ is odd, since - unlike in the nonsupersymmetric 
case - not every superdifferential operator of even, say $2 n$, order has a $(2 n)^{\text {th }}$ root. The general odd order Lax hierarchy was investigated in some detail by Manin and Radul. They showed that the hierarchy was hamiltonian with a supersymmetric analogue of the first Gel'fand-Dickey bracket and that the conserved charges generate the Lax flows relative to this hamiltonian structure.

In order to study the most general even order supersymmetric Lax hierarchy one cannot then start from the SKP hierarchy, but rather on needs to introduce the even order SKP hierarchy (denoted in this paper $\mathrm{SKP}_{2}$ ) which starts from a basic S $\Psi D O$ of the form $D^{2}+\sum_{i \geq-1} A_{i} D^{-i}$ and is supported by the fact (see next section) that the most general Lax operator of order $2 n$ has a unique $n^{\text {th }}$ root. In section 2 we study this hierarchy in some detail and prove its formal integrability. After reviewing the necessary formalism concerning STDO's we introduce the $\mathrm{SKP}_{2}$ hierarchy, construct an infinite number of conservation laws and prove that there are an infinite number of commuting Lax flows. The only feature of the SKP hierarchy missing from $\mathrm{SKP}_{2}$ are the odd flows. However we do not feel that this is an important drawback. In section 3 we study the reductions of $\mathrm{SKP}_{2}$ obtained by imposing that $\Lambda_{-}^{k}=0$. We call them generalized SKdV hierarchies in analogy with the nonsupersymmetric case and anticipating the results of section 4. We prove that these hierarchies are bihamiltonian relative to the supersymmetric analogs of the Gel'fand-Dickey brackets ${ }^{[\mathbf{5}]}$ constructed in [6] . This allows us to prove that the conservation laws obey Lenard-type recursion relations and that they are in involution relative to both Poisson structures. Hence proving the hamiltonian integrability of the hierarchies. In section 4 we consider the simplest example of these reductions and reduce it even further. The resulting hierarchy is identified with that of the supersymmetric extension of the KdV equation (SKdV) introduced in [4] by Manin and Radul. We show that the bihamiltonian nature still persists after reduction; although one of the Poisson structures is now nonlocal. In particular this allows us to prove the hamiltonian integrability of the SKdV hierarchy; a result that was already announced in [7]. Section 5 offers some concluding remarks. 


\section{$\S 2$ EVEN ORDER SKP HIERARCHY}

In this section we define and discuss the basic results associated to the even order SKP hierarchy. We would like to stress that this hierarchy is different from the one considered in [8], which is simply the subhierarchy of the SKP hierarchy introduced by Manin and Radul ${ }^{[4]}$ obtained by taking even powers of the basic SKP operator. The hierarchy of [8] is the specialization of the one here by demanding that the basic even order SKP operator have a square root. As shown in [4] this is not always the case.

In order to define the even order SKP hierarchy, we will consider the space of differential operators on a (1|1) superspace with coordinates $(x, \theta)$. These operators are polynomials in the supercovariant derivative $D=\partial_{\theta}+\theta \partial$ whose coefficients are superfields. The supercovariant derivative obeys $D^{2}=\partial$. We shall call those superfields annihilated by $D$ constants. The basic objects in the (even order) SKP

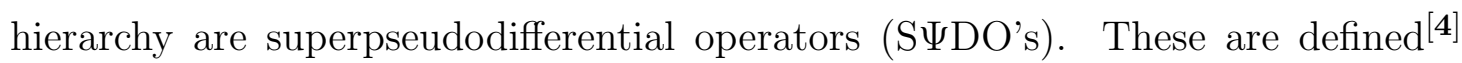
as formal Laurent series in $D^{-1}$ whose coefficients are superfields, where $D^{-1}$ is a formal inverse to $D$. The multiplication of SUDO's is given by the following composition law (for any $n \in \mathbb{Z}$ )

$$
D^{n} \Phi=\sum_{i=0}^{\infty}\left[\begin{array}{c}
n \\
n-i
\end{array}\right](-1)^{|\Phi|(n-i)} \Phi^{[i]} D^{n-i}
$$

for $\Phi$ any superfield and where the superbinomial coefficients are given by

$$
\left[\begin{array}{c}
n \\
n-i
\end{array}\right]= \begin{cases}0 & \text { for } i<0 \text { or }(n, i) \equiv(0,1) \quad(\bmod 2) \\
\left(\begin{array}{c}
{\left[\frac{n}{2}\right]} \\
{\left[\frac{n-i}{2}\right]}
\end{array}\right) & \text { for } i \geq 0 \text { and }(n, i) \not \equiv(0,1) \quad(\bmod 2)\end{cases}
$$


Given a $\operatorname{S\Psi DO} P=\sum p_{i} D^{i}$ we define its super-residue as sres $P=p_{-1}$ and its (Adler) supertrace as $\operatorname{Str} P=\int_{B}$ sres $P$, where $\int_{B}$ is defined as follows. If $f=a+\theta b$, then $\int_{B} f=\int b$, where the precise meaning of integration will depend on the context. It denotes integration over the real line if we take our basic fields to be rapidly decreasing functions; integration over one period if we take them to be periodic functions; or, more abstractly, a linear map annihilating derivatives so that we can "integrate by parts". One can show ${ }^{[4]}$ that the super-residue of a graded commutator is a perfect derivative so that its supertrace vanishes: $\operatorname{Str}[P, Q]=0$, for $[P, Q] \equiv P Q-(-1)^{|P||Q|} Q P$. This then defines a supersymmetric bilinear

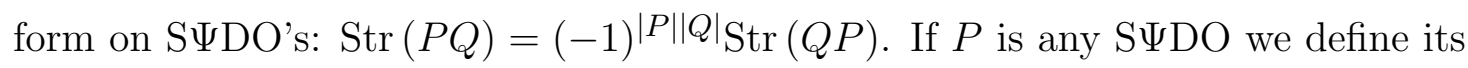
differential part $P_{+}$as the part of $P$ which is polynomial in $D$ (including free terms) and its "integral" part $P_{-}$as simply $P-P_{+}$. It then follows that $\operatorname{Str} P_{ \pm} Q_{ \pm}=0$

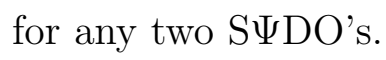

It was proven in [4] that every homogeneous $\mathrm{S} \Psi \mathrm{DO}$ of the form $L=D^{n}+$ $U_{n-1} D^{n-1}+\cdots$ for $n$ odd, has a unique $n^{\text {th }} \operatorname{root} L^{1 / n}=D+\cdots$. For $n$ even, the $n^{\text {th }}$ root need not exist nor be unique; although a unique $(n / 2)^{\text {th }}$ root does exist.

Lemma 2.3. Let $L=D^{2 k}+\sum_{i \leq 2 k-1} U_{i} D^{i}$ be a homogeneous $S \Psi D O$ of even order. Then there exists a unique even $S \Psi D O L^{1 / k}=D^{2}+\cdots$ satisfying $\left(L^{1 / k}\right)^{k}=L$ (hence the name). Furthermore, the coefficients of $L^{1 / k}$ are differential polynomials in the coefficients of $L$.

Proof: Let $\Lambda=D^{2}+\sum_{i=-1}^{\infty} A_{i} D^{-i}$. Computing one finds that

$$
\Lambda^{k}=D^{2 k}+k A_{-1} D^{2 k-1}+O\left(D^{2 k-2}\right)
$$

whence, if we take $A_{-1}=\frac{1}{k} U_{2 k-1}, \Lambda^{k}-L=O\left(D^{2 k-2}\right)$. Suppose now that $A_{-1}, A_{0}, \ldots, A_{n}$, differential polynomials ${ }^{1}$ in $L$, have been found so that $\Lambda^{k}-L=$

1 By differential polynomials in an operator we will always mean, of course, differential polynomials in its coefficients. 
$O\left(D^{2 k-n-3}\right)$. A brief calculation shows that the term of order $D^{2 k-n-3}$ is given by $k A_{n+1}-X$, where $X$ is some differential polynomial in the $U_{i}$ and in the $A_{i \leq n}$ hence in the $U_{i}$. Therefore setting $A_{n+1}=\frac{1}{k} X$, allows to extend the induction hypothesis one step further. It is clear that $L^{1 / k}=\Lambda$ is the desired $k^{\text {th }}$ root.

We are interested in constructing Lax-type evolution equations of the form

$$
\frac{\partial L}{\partial t}=[P, L]
$$

where

$$
L=D^{2 k}+\sum_{i=0}^{2 k-1} U_{i} D^{i}
$$

is a homogeneous superdifferential operator and $P$ is a also homogeneous superdifferential operators whose coefficients are differential polynomials in $L$. The following result motivates the introduction of the even order SKP hierarchy.

Proposition 2.7. $L$ satisfies (2.5) if and only if its $k^{\text {th }}$ root $L^{1 / k}$ does. That is,

$$
\frac{\partial L}{\partial t}=[P, L] \quad \Leftrightarrow \quad \frac{\partial L^{1 / k}}{\partial t}=\left[P, L^{1 / k}\right]
$$

Proof: Write the Lax equation as $\left[\frac{\partial}{\partial t}-P, L\right]=0$. It is then clear that $\left[\frac{\partial}{\partial t}-\right.$ $\left.P, L^{1 / k}\right]=0 \Rightarrow\left[\frac{\partial}{\partial t}-P, L\right]=0$, since $\left[\frac{\partial}{\partial t}-P, \cdot\right]$ is a derivation and $L=\left(L^{1 / k}\right)^{k}$. Conversely, let $\left[\frac{\partial}{\partial t}-P, L^{1 / k}\right]=\sum_{i \leq N} A_{i} D^{i}$. Then

$$
\begin{aligned}
0 & =\left[\frac{\partial}{\partial t}-P,\left(L^{1 / k}\right)^{k}\right] \\
& =\sum_{j=0}^{k-1} L^{j / k}\left[\frac{\partial}{\partial t}-P, L^{1 / k}\right] L^{(k-j-1) / k} \\
& =k A_{N} D^{N+2 k-2}+O\left(D^{N+2 k-3}\right)
\end{aligned}
$$

whence $A_{N}=0$. Thus, $\left[\frac{\partial}{\partial t}-P, L^{1 / k}\right]=0$. 
From now on until the end of this section we will investigate the possible Lax-type evolution equations of a general even $\mathrm{S} \Psi \mathrm{DO}$ of the form $\Lambda=D^{2}+\cdots$. The resulting hierarchy is the even order SKP hierarchy $\left(\mathrm{SKP}_{2}\right)$. By imposing the constraint $\left(\Lambda^{k}\right)_{-}=0$, we will obtain other hierarchies for the even order supersymmetric Lax operators as reductions of $\mathrm{SKP}_{2}$. These reductions will be the main topic of the following section.

We must first determine the superdifferential operators $P$ for which the equation

$$
\frac{\partial \Lambda}{\partial t}=[P, \Lambda]
$$

is a consistent (local) evolution equation, i.e. , for which homogeneous differential operators $P$, whose coefficients are differential polynomials in $\Lambda$, is the right hand side of (2.9) a STDO of order at most one. Let us denote the space of such $P$ by

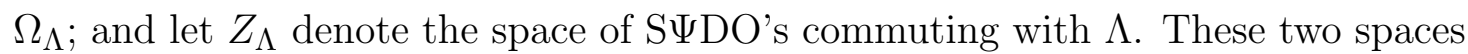
are related as follows.

Lemma 2.10. If $M \in Z_{\Lambda}$, then $M_{+} \in \Omega_{\Lambda}$.

Proof: If $M \in Z_{\Lambda}$ then $\left[M_{+}, L\right]=\left[L, M_{-}\right]$, whence $\left[M_{+}, L\right]$ has order at most 1 .

Therefore it is important to characterize the centralizer $Z_{\Lambda}$ of $\Lambda$. The following Proposition does just that.

Proposition 2.11. As a vector space over the constants, $Z_{\Lambda}$ is spanned by the powers $\Lambda^{n}$, for $n \in \mathbb{Z}$. 
Proof: Let $\Lambda=D^{2}+\sum_{-1}^{\infty} A_{i} D^{-i}$ and $M \in Z_{\Lambda}$. Let us first assume that $M=$ $\sum_{j \leq 2 n+1} B_{j} D^{j}$ has odd order. Then order by order in $D$, the condition $[M, \Lambda]=0$ becomes an infinite number of equations for the coefficients of $M$. The first two such equations are

$$
A_{-1} B_{2 n+1}=0
$$

and

$$
B_{2 n+1}^{\prime \prime}-\left(A_{-1} B_{2 n+1}\right)^{\prime}=0
$$

where we denote the action of $D$ by '. Using the first equation in the second, we find that $B_{2 n+1}$ is a constant and back into the first equation that the constant has to be zero. Thus we find that $Z_{\Lambda}$ consists only of operators of even order $M=\sum_{j \leq 2 n} B_{j} D^{j}$. In this case the first two equations coming from demanding $[M, \Lambda]=0$ are

$$
B_{2 n}^{\prime \prime}+A_{-1} B_{2 n}^{\prime}+2 B_{2 n-1} A_{-1}=0
$$

and

$$
B_{2 n-1}^{\prime \prime}-\left(A_{-1} B_{2 n-1}\right)^{\prime}-n B_{2 n} A_{-1}^{\prime \prime}=0
$$

It is not hard to see that if we demand that the $B_{j}$ be differential polynomials of the $A_{i}$, then the only solution is $B_{2 n}=\lambda, B_{2 n-1}=n A_{-1} \lambda$, for $\lambda$ some constant. In fact, from (2.14) we can formally solve for $B_{2 n}$ as follows

$$
B_{2 n}=-2 D^{-1}\left(D+A_{-1}\right)^{-1} A_{-1} B_{2 n-1},
$$

where we have assumed that $B_{2 n}$ is not a constant so that we can invert $D$. Substituting this into (2.15) we find an equation for $B_{2 n-1}$ of the form $P B_{2 n-1}=0$, with $P$ a S $\Psi D O$ with leading term $D^{2}$. Since such a $P$ is invertible, we find that $B_{2 n-1}=0$ and thus that $B_{2 n}=0$; whence the only nontrivial solution is $B_{2 n}$ constant. Therefore, $M-\lambda \Lambda^{n} \in Z_{\Lambda}$ is an operator of smaller order than $M$. Applying the argument repeatedly we are done.

From this we can immediately characterize $\Omega_{\Lambda}$. 
Proposition 2.17. The most general element of $\Omega_{\Lambda}$ is given by a linear combination with constant coefficients of $\Lambda_{+}^{n}$, for $n \in \mathbb{N}$ and by any superdifferential operator of the form $f D^{2}+g D+h$, where $h$ is an arbitrary differential polynomial of $\Lambda$ and $f$ and $g$ are differential polynomials of $\Lambda$ subject to the condition

$$
f^{\prime \prime}+A_{-1} f^{\prime}+2 g A_{-1}=0
$$

where $A_{-1}$ is the coefficient of $D$ in $\Lambda$.

Proof: Let $\Lambda$ be as in the above proof, and let $M \in \Omega_{\Lambda}$ have order $>2$. Then by the same arguments as in the previous proof, we find that the order must be even, say $2 n$, and its leading coefficient be a constant, say $c_{n}$. Then $M-c_{n} \Lambda_{+}^{n} \in \Omega_{\Lambda}$ has order at most $2 n-1$. Continuing in this way we find that we can subtract constant linear combinations of the $\Lambda_{+}^{i}$ until we are left with an operator of order 2. For this case the argument of the previous proof does not work, since we only have the analogous of equation (2.14) (for $n=1$ ) but not the one of equation (2.15) , since the term of order $D$ in $[M, \Lambda]$ does not have to vanish. Therefore, we are left with a general second order superdifferential operator $f D^{2}+g D+h$ with $f, g$, and $h$ differential polynomials in $\Lambda$ subject to the relation (2.14) for $n=1$, which is precisely $(2.18)$.

Remark 2.19. This result should be contrasted to the similar result for KP (see, e.g. , [2] ) where $\Omega_{L_{K P}}$ is generated as a vector space over the constants by the functions together with the differential part of the powers of $L_{K P}$. In that case one can disregard the flows generated by the functions by demanding that the free coefficient in $L_{K P}$ vanish, since those flows do not preserve that constraint. Analogously, in our case, we could restrict ourselves to the first two coefficients in $\Lambda$ being zero, in which case these "exotic" flows would not be present either. We will not do this here but, rather, we will disregard those flows altogether and consider instead the subhierarchy of flows are generated by the linear combinations of the operators $\Lambda_{+}^{n}$ with constant coefficients. This restriction may seem unwarranted from the point of view of the $\mathrm{SKP}_{2}$ hierarchy, but it will turn out that the flows induced on the reduced hierarchies by these exotic Lax operators will not be hamiltonian with respect to the supersymmetric version of the Gel'fand-Dickey brackets. 
Let us then introduce an infinite number of "time" variables $t_{n}$ for $n \in \mathbb{N}$ and define the following flows associated to them:

$$
\frac{\partial \Lambda}{\partial t_{n}}=\left[\Lambda_{+}^{n}, \Lambda\right]
$$

According to the following Proposition these flows commute:

Proposition 2.21. For all $m, n \in \mathbb{N}$,

$$
\frac{\partial^{2} \Lambda}{\partial t_{m} \partial t_{n}}=\frac{\partial^{2} \Lambda}{\partial t_{n} \partial t_{m}} .
$$

Proof:

$$
\begin{aligned}
\frac{\partial^{2} \Lambda}{\partial t_{m} \partial t_{n}} & =\frac{\partial}{\partial t_{m}}\left[\Lambda_{+}^{n}, \Lambda\right] \\
& =\left[\left(\frac{\partial \Lambda^{n}}{\partial t_{m}}\right)_{+}, \Lambda\right]+\left[\Lambda_{+}^{n},\left[\Lambda_{+}^{m}, \Lambda\right]\right] \\
& =\left[\left[\Lambda_{+}^{m}, \Lambda^{n}\right]_{+}, \Lambda\right]+\left[\Lambda_{+}^{n},\left[\Lambda_{+}^{m}, \Lambda\right]\right] \\
& =\left[\left[\Lambda_{+}^{m}, \Lambda^{n}\right]_{+}, \Lambda\right]+\left[\Lambda_{+}^{m},\left[\Lambda_{+}^{n}, \Lambda\right]\right]+\left[\left[\Lambda_{+}^{n}, \Lambda_{+}^{m}\right], \Lambda\right] \\
& =\left[\left[\Lambda_{+}^{m}, \Lambda_{-}^{n}\right]_{+}, \Lambda\right]+\left[\Lambda_{+}^{m},\left[\Lambda_{+}^{n}, \Lambda\right]\right] \\
& =\left[\left[\Lambda^{m}, \Lambda_{-}^{n}\right]_{+}, \Lambda\right]+\left[\Lambda_{+}^{m},\left[\Lambda_{+}^{n}, \Lambda\right]\right] \\
& =\left[\left[\Lambda_{+}^{n}, \Lambda^{m}\right]_{+}, \Lambda\right]+\left[\Lambda_{+}^{m},\left[\Lambda_{+}^{n}, \Lambda\right]\right] \\
& =\frac{\partial^{2} \Lambda}{\partial t_{n} \partial t_{m}} .
\end{aligned}
$$

The $\mathrm{SKP}_{2}$ hierarchy has an infinite number of nontrivial independent polynomial conserved quantities. Define $H_{n}=\frac{1}{n} \operatorname{Str} \Lambda^{n}$, for every $n \in \mathbb{N}$. It is clear that they are the integrals of polynomial densities and, it follows at once from the fact that the supertrace annihilates (graded) commutators, that these quantities are conserved. Furthermore they are non-trivial and linearly independent as the next proposition shows. 
Proposition 2.23. The $H_{n}$ for $n \in \mathbb{N}$ form an infinite set of non-trivial, linearly independent, polynomial conserved charges for the $\mathrm{SKP}_{2}$ hierarchy.

Proof: It is obvious that these charges are polynomial. Independence follows at once from the fact that one can grade the differential polynomials in such a way that these charges all have different degree. Indeed, let us define the following

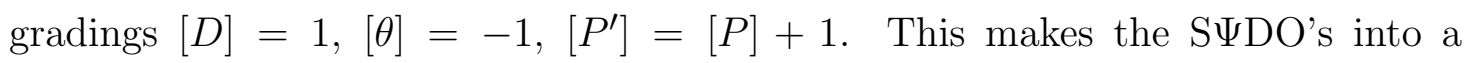
graded ring such that its reduction modulo 2 coincides with the natural $\mathbb{Z}_{2}$ grading. Now assign degrees to the coefficients of $\Lambda$ in such a way that $[\Lambda]=2$. It then follows that $\left[H_{n}\right]=2 n$ and hence that they are independent. Now let $\Lambda=D^{2}+$ $\sum_{i=-1}^{\infty} A_{i} D^{-i}$. It is easy to see that in the expression for the super-residue of $\Lambda^{n}$ there always appears a linear term $A_{2 n-1}$. If $H_{n}=0$ for some $n$, it would then imply a differential relation between the $A_{i}$; namely that $A_{2 n-1}$ is given, up to a perfect derivative, by a differential polynomial of the $A_{i<2 n-1}$, which contradicts the assumption that the $A_{i}$ are differentially independent.

This proves the formal integrability of the $\mathrm{SKP}_{2}$ hierarchy. It is noteworthy that all the conserved charges are even under the $\mathbb{Z}_{2}$ grading.

\section{$\S 3$ EVEN ORDER GENERALIZED SKDV HIERARCHIES}

In this section we study the family of reductions of the $\mathrm{SKP}_{2}$ hierarchy defined as follows. Fix a positive integer $k \in \mathbb{N}$ and impose the constraint that the operator $L \equiv \Lambda^{k}$ be differential, that is, $\left(\Lambda^{k}\right)_{-}=0$ and such that $k$ is the smallest such number for which this is true. The $\mathrm{SKP}_{2}$ flows clearly induce flows

$$
\begin{aligned}
\frac{\partial L}{\partial t_{n}} & =\left[\Lambda_{+}^{n}, L\right] . \\
& -11-
\end{aligned}
$$


Notice, however, that for $n$ a multiple of $k$, these flows are trivial. Since the $k^{\text {th }}$ root of $L$ is a specialization of the general $\mathrm{SKP}_{2}$ operator treated in the last section, a lot of results can be immediately exported to this case. In particular, the characterization of the possible evolution equations, the commutativity of the flows, and the existence of the infinite number of conserved charges follow from the analogous results for the unreduced hierarchy. Only the nontriviality of the conserved charges needs a new argument. We will prove this after introducing a bihamiltonian structure for the reduced hierarchy. We call this hierarchy the generalized $k^{\text {th }}$ order super Korteweg-de Vries (SKdV) hierarchy or, to make obvious the fact that this is a reduction of the even order SKP hierarchy, $\mathrm{SKP}_{2}^{(k)}$.

Let $L$ be as in (2.6). Its $k^{\text {th }}$ root $L^{1 / k}$, which exists by Lemma 2.3 , is a spe-

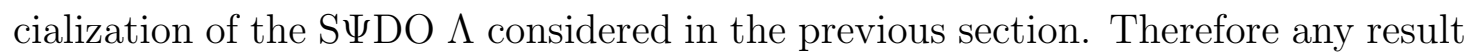
which does not use the fact that the coefficients of $\Lambda$ are independent immediately holds. In fact, this observation, together with Proposition 2.7, allows us to prove the following results as we did in the previous section. We shall therefore omit their proofs here. Define $Z_{L}$ and $\Omega_{L}$ in the obvious way. Then we have the following three results analogous to Lemma 2.10, Proposition 2.11, and Proposition 2.17 :

Lemma 3.2. If $M \in Z_{L}$, then $M_{+} \in \Omega_{L}$.

Proposition 3.3. As a vector space over the constants, $Z_{L}$ is spanned by the powers $L^{n / k}$, for $n \in \mathbb{Z}$.

Proposition 3.4. The most general element of $\Omega_{L}$ is given by a linear combination with constant coefficients of $L_{+}^{(n / k)}$, for $n \in \mathbb{N}$ and by any superdifferential operator of the form $f D^{2}+g D+h$, where $h$ is an arbitrary differential polynomial of $L$ and $f$ and $g$ are differential polynomials of $L$ subject to the condition

$$
k f^{\prime \prime}+U_{2 k-1} f^{\prime}+2 g U_{2 k-1}=0 .
$$


As in the unreduced hierarchy ( $c f$. Remark 2.19 ), we will disregard the flows generated by the Lax operators which are not constant linear combinations of the $L_{+}^{(n / k)}$. We will, thus, associate a time $t_{n}$ with each generator $L_{+}^{n / k}$ of $\Omega_{L}$ as follows:

$$
\frac{\partial L}{\partial t_{n}}=\left[L_{+}^{n / k}, L\right]
$$

Of course, those times $t_{n}$ with $n$ a multiple of $k$ will yield trivial evolution equations and can be disregarded. In exactly the same way we proved Proposition 2.21 we obtain that these flows also commute.

Proposition 3.7. For all $m, n \in \mathbb{N}$,

$$
\frac{\partial^{2} L}{\partial t_{m} \partial t_{n}}=\frac{\partial^{2} L}{\partial t_{n} \partial t_{m}}
$$

This hierarchy possesses an infinite number of polynomial conserved quantities. Let $n \in \mathbb{N}$ and define $H_{n} \equiv \frac{k}{n}$ Str $L^{n / k}$. Most of the analogous result to Proposition 2.23 can still be proven in the same way, except for the nontriviality of the conserved charges, since that part of the proof used the fact that the coefficients of $\Lambda$ were independent. It follows from the proof of Lemma 2.3 that only the first $k$ coefficients of $L^{1 / k}$ are independent in our case. Since the grading argument used to prove Proposition 2.23 still goes through, the only thing that could go wrong is for a charge to be identically zero. This already happens to the charges $H_{n}$ with $n$ a multiple of $k$. We will see that, in fact, these are the only ones for which this happens. But in order to prove this we will use the fact that the evolution equations are hamiltonian, a result which is interesting in its own right and which we now discuss.

In order to define a Poisson structure in the space $M$ of Lax operators of the form (2.6) , we need to define several formal geometric objects on $M$ : functions, vector fields, 1-forms, ... This is done in detail, for example, in [9] and thus we only briefly list the results here. 
We will take as our functions objects of the form

$$
F[L]=\int_{B} f(U)
$$

where $f(U)$ is a homogeneous differential polynomial of the $U_{i}$. Vector fields are parametrized by infinitesimal deformations $L \mapsto L+\epsilon A$ where $A=\sum A_{l} D^{l}$ is a homogeneous differential operator of order at most $2 k-1$. We denote the space of such operators by $S_{2 k}$. To such an operator $A \in S_{2 k}$ we associate a vector field $D_{A}$ as follows. If $F=\int_{B} f$ is a function then

$$
\begin{aligned}
D_{A} F & \left.\equiv \frac{d}{d \epsilon} F[L+\epsilon A]\right|_{\epsilon=0} \\
& =(-1)^{|A|} \int_{B} \sum_{j=0}^{2 k-1} \sum_{i=0}^{\infty}(-1)^{|A| i} A_{j}^{[i]} \frac{\partial f}{\partial U_{j}^{[i]}},
\end{aligned}
$$

with $U_{j}^{[i]}=\left(D^{i} U_{j}\right)$ and the same for $A_{j}^{[i]}$. Integrating by parts we can write this as

$$
D_{A} F=(-1)^{|A|} \int_{B} \sum_{j=0}^{2 k-1} A_{j} \frac{\delta F}{\delta U_{j}}
$$

where the Euler variational derivative is given by

$$
\frac{\delta F}{\delta U_{j}}=\sum_{i=0}^{\infty}(-1)^{\left|U_{j}\right| i+i(i+1) / 2} D^{i} \frac{\partial f}{\partial U_{j}^{[i]}}
$$

We define 1-forms as the space $S_{2 k}^{*}$ of STDO's of the form $X=\sum_{i=0}^{2 k-1} D^{-i-1} X_{i}$, whose pairing with a vector field $D_{A}$, with $A=\sum A_{i} D^{i}$, is given by

$$
\left(D_{A}, X\right) \equiv(-1)^{|A|+|X|+1} \operatorname{Str}(A X)=(-1)^{|A|} \int_{B} \sum_{i=0}^{2 k-1}(-1)^{i} A_{i} X_{i}
$$


which is nondegenerate. The choice of signs has been made to avoid undesirable signs later on. Given a function $F=\int_{B} f$ we define its gradient $d F$ by

$$
\left(D_{A}, d F\right)=D_{A} F
$$

whence, comparing with (3.11), yields

$$
d F=\sum_{i=0}^{2 k-1}(-1)^{i} D^{-i-1} \frac{\delta F}{\delta U_{i}}
$$

Therefore the gradient of a function is a 1-form, as expected.

To define Poisson brackets on the space of supersymmetric Lax operators, we start by defining a map $J: S_{2 k}^{*} \rightarrow S_{2 k}$ in such a way that the Poisson bracket of two functions $F$ and $G$ is given by

$$
\{F, G\}=D_{J(d F)} G=\left(D_{J(d F)}, d G\right)=(-1)^{|J|+|F|+|G|+1} \operatorname{Str}(J(d F) d G) .
$$

Demanding that the Poisson brackets defined by $J$ obey the correct (anti)symmetry properties and the Jacobi identity imposes strong restrictions on the allowed maps $J$. Maps obeying these conditions are often called "hamiltonian". It was proven in [6] that the map

$$
\begin{aligned}
J: S_{2 k}^{*} & \rightarrow S_{2 k} \\
X & \mapsto(L X)_{+} L-L(X L)_{+}
\end{aligned}
$$

is hamiltonian. The proof in [6] employed a supersymmetric version of the Miura transformation and, in fact, established a supersymmetric version of the celebrated Kupershmidt-Wilson theorem $\left.{ }^{[\mathbf{1 0}}\right]$ along the lines of Dickey ${ }^{[\mathbf{1 1}]}$. In [9] , a purely combinatorial proof of the hamiltonian property of $J$ was given which yields, $m u$ tatis mutandis, a proof of the following result. 
Proposition 3.18. Let $z$ be a parameter and define $\hat{L} \equiv L-z^{k}$. Then the map $J_{z}: S_{2 k}^{*} \rightarrow S_{2 k}$ defined by

$$
J_{z}(X)=(\hat{L} X)_{+} \hat{L}-\hat{L}(X \hat{L})_{+}
$$

is hamiltonian.

Making the dependence on the parameter $z$ manifest, we find that $J_{z}$ decomposes as $J_{z}=J_{0}-z^{k} J_{\infty}$, where $J_{0}$ is given by (3.17) and $J_{\infty}$ is given by

$$
J_{\infty}(X)=[L, X]_{+}
$$

This defines a one-parameter family of Poisson brackets

$$
\begin{aligned}
\{F, G\}_{z} & =-(-1)^{|F|+|G|} \operatorname{Str}\left(J_{z}(d F) d G\right), \\
& =\{F, G\}_{0}-z^{k}\{F, G\}_{\infty},
\end{aligned}
$$

where

$$
\{F, G\}_{0}=-(-1)^{|F|+|G|} \operatorname{Str}\left((L d F)_{+} L d G-L(d F L)_{+} d G\right)
$$

and

$$
\{F, G\}_{\infty}=-(-1)^{|F|+|G|} \operatorname{Str}\left([L, d F]_{+} d G\right)
$$

are the supersymmetric analogs of the Gel'fand-Dickey brackets. The latter Poisson bracket can be rewritten as

$$
\{F, G\}_{\infty}=-(-1)^{|F|+|G|} \operatorname{Str}(L[d F, d G])
$$

which is nothing but the Kirillov-Kostant Poisson structure on the coadjoint orbit of $L$ under the supersymmetric Volterra group. 
Suppose now that $H$ is a function on $M$. Given a hamiltonian map $J$ and associated Poisson bracket $\{$,$\} , one can define a flow associated to H$ as follows:

$$
\frac{\partial L}{\partial t}=J(d H)
$$

or, equivalently,

$$
\frac{\partial F}{\partial t}=\{H, F\}
$$

for $F$ any function on $M$. If for $J$ we take $J_{\infty}$, then from (3.20) we can write this flow in a way which suggests a Lax-type equation,

$$
\frac{\partial L}{\partial t}=J_{\infty}(d H)=[L, d H]_{+}
$$

The following lemma pursues this suggestion.

Lemma 3.29. Let $H_{n} \equiv-\frac{k}{n} \operatorname{Str} L^{n / k}$. Then its gradient is given simply by

$$
d H_{n}=L_{-}^{\frac{n}{k}-1} \bmod D^{-2 k-1} .
$$

Proof: If $A$ is any vector field, the directional derivative of $H_{n}$ in the direction specified by $A$ is given by

$$
\begin{aligned}
D_{A} H_{n}[L] & =\left.\frac{d}{d \epsilon} H_{n}[L+\epsilon A]\right|_{\epsilon=0} \\
& =-\left.\frac{k}{n} \frac{d}{d \epsilon} \operatorname{Str}(L+\epsilon A)^{n / k}\right|_{\epsilon=0} \\
& =-(-1)^{|A|} \operatorname{Str} A L^{\frac{n}{k}-1}
\end{aligned}
$$

whence, after comparing with (3.14), the lemma follows.

Together with (3.28) and (3.6), Lemma 3.29 immediately yields 
Proposition 3.31. With respect to the Poisson structure defined by $J_{\infty}$, the conserved charge $H_{n+k}$ is the hamiltonian generating the flow along the time $t_{n}$. In other words,

$$
\frac{\partial L}{\partial t_{n}}=J_{\infty}\left(d H_{n+k}\right)
$$

Proof: From Lemma 3.29 and (3.20) we find

$$
\begin{aligned}
J_{\infty}\left(d H_{n+k}\right) & =\left[L, L_{-}^{n / k}\right]_{+} \\
& =\left[L_{+}^{n / k}, L\right],
\end{aligned}
$$

which by (3.6) is precisely $\frac{\partial L}{\partial t_{n}}$.

Remark 3.33. It is possible to show that those Lax operators in $\Omega_{L}$ which are not just constant linear combinations of $L_{+}^{(n / k)}$ generate flows which are not hamiltonian with respect to the Poisson structure $J_{\infty}$. We immediately see that none of the flows which are hamiltonian with respect to $J_{\infty}$ evolve $U_{2 k-1}$ in time, since, for any $H$, the order of $[L, d H]_{+}$is at most $2 k-2$. This already means that for an exotic Lax operator to generate a hamiltonian flow with respect to $J_{\infty}$, it must have the form $c L_{+}^{1 / k}+h$, where $c$ is some constant and $h$ is an arbitrary differential polynomial in $L$. Moreover, a calculation then shows that $h$ cannot generate a hamiltonian flow with respect to $J_{\infty}$, unless it is a constant, in which case the flow is trivial.

From the hamiltonian nature of the flows we can already prove the nontriviality of the conserved charges $H_{n}$ for $n$ not a multiple of $k$.

Corollary 3.34. The $H_{n}$, for $n \in \mathbb{N}$ not a multiple of $k$, form an infinite set of nontrivial, linearly independent, polynomial conserved charges for the $\mathrm{SKP}_{2}^{(k)}$ hierarchy. 
Proof: That the charges are conserved follows from Proposition 2.7 and the fact that the supertrace vanishes on (graded) commutators. Now, the charges are obviously polynomial and the proof of linear independence is identical to that of the similar statement ( $c f$. Proposition 2.23 ) for the unreduced hierarchy. Only the proof of nontriviality changes. If $H_{n+k}=0$, its associated flow is trivial; whence $\frac{\partial L}{\partial t_{n}}=0$. According to (3.6) this means that $L_{+}^{n / k}$ commutes with $L$. But in Proposition 3.3 , we characterized those elements as linear combinations with constant coefficients of the $L^{i / k}$. By comparing the degree of homogeneity and the leading term, we conclude that $L_{+}^{n / k}$ has to be $L^{n / k}$ itself. Thus, $L_{-}^{n / k}=0$. Taking the supertrace we see that $H_{n}=0$. Writing $n=s k+r$, with $0 \leq r<k$, we eventually find that $\left(L^{1 / k}\right)_{-}^{r}=0$. But by the definition of the hierarchy, this is not true for any $r<k$ unless $r=0$.

The rest of the section is devoted to the bihamiltonian structure of the $\mathrm{SKP}_{2}^{(k)}$ hierarchy. It turns out that the evolution equations of this hierarchy are hamiltonian with respect to $J_{0}$ as well. This will follow from the Lenard relations for the conserved charges. Let us introduce the notion of a "basic resolvent". Let $z$ be the parameter introduced in Proposition 3.18 and define the following formal series

$$
R(z)=\sum_{n \in \mathbb{Z}} z^{-n-k} L_{-}^{n / k}
$$

For our purposes, the most important property of $R(z)$ is the following:

Lemma 3.36. Let $\hat{L} \equiv L-z^{k}$. Then both $\hat{L} R(z)$ and $R(z) \hat{L}$ are differential operators.

Proof: Computing one finds

$$
\begin{aligned}
\left(L-z^{k}\right) R(z) & =\sum_{n \in \mathbb{Z}}\left[z^{-n-k} L L_{-}^{n / k}-z^{-n} L_{-}^{n / k}\right] \\
& =\sum_{n \in \mathbb{Z}} z^{-n-k}\left[L L_{-}^{n / k}-\left(L L^{n / k}\right)_{-}\right] .
\end{aligned}
$$

$\operatorname{But}\left[L L_{-}^{n / k}-\left(L L^{n / k}\right)_{-}\right]_{-}=\left[L L_{-}^{n / k}-L L^{n / k}\right]_{-}=-\left[L L_{+}^{n / k}\right]_{-}=0$. The second statement is proven in a similar fashion. 
This immediately yields a recursive relation between the gradients of the conserved charges.

Proposition 3.37. (Lenard relations) For all $n \in \mathbb{N}$,

$$
J_{\infty}\left(d H_{n+k}\right)=J_{0}\left(d H_{n}\right) .
$$

Proof: From Lemma 3.36 we have that $(\hat{L} R(z))_{+} \hat{L}-\hat{L}(R(z) \hat{L})_{+}=0$, whence $J_{0}(R(z))=J_{\infty}\left(z^{k} R(z)\right)$. Comparing order by order in $z$, and using Lemma 3.29 , we obtain the desired relations.

In particular this implies that the $\mathrm{SKP}_{2}^{(k)}$ hierarchy is also hamiltonian with respect to the Poisson structure defined by $J_{0}$, since the flow along the time $t_{n}$ is generated by $H_{n}$ relative to this Poisson structure.

Proposition 3.39. The conserved charges $H_{n}$ are in involution with respect to both Poisson structures $J_{0}$ and $J_{\infty}$ and, hence, relative to the one parameter family of Poisson brackets $\{,\}_{z}$.

Proof: For all $m, n \in \mathbb{N}, \frac{\partial H_{n}}{\partial t_{m}}=0$. But $\frac{\partial}{\partial t_{m}}$ is the hamiltonian flow generated by $H_{m}$ relative to $J_{0}$ and by $H_{m+k}$ relative to $J_{\infty}$. Thus, for all $m, n \in \mathbb{N}$,

$$
\left\{H_{n}, H_{m}\right\}_{0}=\left\{H_{n}, H_{m}\right\}_{\infty}=0
$$

This, by the way, gives a new proof for Proposition 3.7 . 


\section{$\S 4$ AN EXPLICIT EXAMPLE: THE SKDV HIERARCHY}

In this section we study a reduction of the $\mathrm{SKP}_{2}^{(2)}$ hierarchy. Denoting the Lax operator of the $\operatorname{SKP}_{2}^{(2)}$ by $D^{4}+U_{3} D^{3}+U_{2} D^{2}+U_{1} D+U_{0}$, we will consider the reduction obtained by setting $U_{0}=U_{2}=U_{3}=0$. A more invariant description of this reduction is obtained by noticing that the reduced operator is the unique superdifferential operator of order 4 satisfying ${ }^{2}$

$$
L^{*}=D L D^{-1}
$$

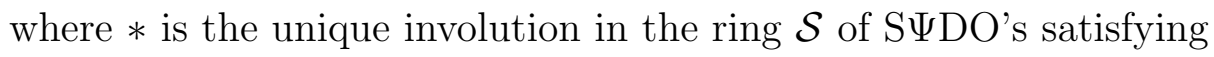

(a) $D^{*}=-D$,

(b) $f^{*}=f$, for any differential polynomial $f$; and,

(c) $(P Q)^{*}=(-1)^{|P||Q|} Q^{*} P^{*}$, for all homogeneous $P, Q \in \mathcal{S}$.

The proof of the following Proposition is routine.

Proposition 4.2. The involution $*$ enjoys the following additional properties:

(1) If $P \in \mathcal{S}$ is homogeneous and invertible, $\left(P^{-1}\right)^{*}=(-1)^{|P|}\left(P^{*}\right)^{-1}$.

(2) For all $p \in \mathbb{Z},\left(D^{p}\right)^{*}=(-1)^{\frac{p(p+1)}{2}} D^{p}$.

(3) For all $P \in \mathcal{S},\left(P_{ \pm}\right)^{*}=\left(P^{*}\right)_{ \pm}$.

(4) For all $P \in \mathcal{S}$, sres $P^{*}=\operatorname{sres} P$ (in particular, $\operatorname{Str} P^{*}=\operatorname{Str} P$ ).

Remarkably all but the exotic flows obtained in the last section survive this reduction. We show this in three steps. First we determine the centralizer of the reduced operator.

2 We could equally well consider the reduction $L^{*}=D^{-1} L D$, which consists in setting $U_{3}=U_{2}=U_{0}+U_{1}^{\prime}=0$. Both reductions yield the same integrable hierarchy. 
Proposition 4.3. As a vector space over the constants, $Z_{L}$ is spanned by the powers $L^{n / k}$, for $n \in \mathbb{Z}$.

Proof: Suppose $M=\sum_{j \leq 2 n} B_{j} D^{j} \in Z_{L}$. Then the first equation in $[M, L]=0$ (i.e. , setting to zero the coefficient at order $2 n+2$ ) is $B_{2 n}^{\prime \prime}=0$, which implies that $B_{2 n}$ is a constant. Therefore $\widetilde{M}=M-B_{2 n} L^{n / 2}$ belongs to $Z_{L}$ and has order $2 n-1$. Let $\widetilde{M}=\sum_{j \leq 2 n-1} \widetilde{B}_{j} D^{j}$. Then the first two equations in $[\widetilde{M}, L]=0$ are obtained by setting to zero the coefficients at orders $2 n+1$ and $2 n$ :

$$
\widetilde{B}_{2 n-1}^{\prime \prime}=0
$$

and

$$
\widetilde{B}_{2 n-2}+\widetilde{B}_{2 n-1} U=0
$$

The first equation says that $\widetilde{B}_{2 n-1}$ is a constant, whereas the second says that $U$ times a constant is a total derivative, which is absurd unless the constant is zero. Hence $\widetilde{B}_{2 n-1}=0$. By induction we obtain the desired result.

Let us define $\Omega_{L}$ as those differential operators with coefficients in the differential polynomials of $L$ whose commutator with $L$ is of order at most 1 . This extra condition leaves behind the exotic flows obtained in the previous section.

Proposition 4.4. As a vector space over the constants, $\Omega_{L}$ is spanned by the powers $L^{n / k}$, for $n \in \mathbb{Z}$.

Proof: The proof follows similar lines to the previous one. If $M=\sum_{0}^{2 n} B_{j} D^{j} \in$ $\Omega_{L}$ and $n \geq 0$ then we find that $B_{2 n}$ is a constant and we can subtract $B_{2 n} L_{+}^{n / 2}$. If, on the other hand, $M$ has odd order then it follows as before that the leading coefficient actually has to vanish.

As we have defined it, however, belonging to $\Omega_{L}$ is not a sufficient criterion for an operator to induce a consistent evolution equation. We should also make sure that the free term of $L$ does not evolve in time. In principle this could get rid of some flows but, remarkably, it doesn't. 
Proposition 4.5. For all operators $P \in \Omega_{L}$, the Lax equation

$$
\frac{\partial L}{\partial t}=[P, L]
$$

is a consistent evolution equation.

Proof: By linearity and the previous Proposition we may (and will) take $P=$ $L_{+}^{n / 2}$. Since $L$ has no free term, we have to show that $\left[L_{+}^{n / 2}, L\right]$ has no free term. For this same reason this is equivalent to $L_{+}^{n / 2}$ (equivalently $L^{n / 2}$ ) having no free

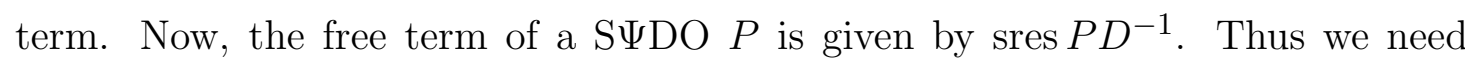
to show that sres $L^{n / 2} D^{-1}$ vanishes. As will be shown in the following lemma, $\left(L^{n / 2}\right)^{*}=(-1)^{n} D L^{n / 2} D^{-1}$. Then using Proposition 4.2 (4), we find

$$
\begin{aligned}
\operatorname{sres} L^{n / 2} D^{-1} & =\operatorname{sres}\left(L^{n / 2} D^{-1}\right)^{*} \\
& =\operatorname{sres} D^{-1}\left(L^{n / 2}\right)^{*} \\
& =(-1)^{n} \operatorname{sres} L^{n / 2} D^{-1},
\end{aligned}
$$

which vanishes for $n$ odd. For $n$ even, $L^{n / 2}$ is an integer power of $L$ and hence has no free term since $L$ has none.

Lemma 4.6. $\left(L^{n / 2}\right)^{*}=(-1)^{n} D L^{n / 2} D^{-1}$.

Proof: From $\left(D L^{1 / 2} D^{-1}\right)^{2}=D L D^{-1}=L^{*}$ it follows that $D L^{1 / 2} D^{-1}$ is equal to $\left(L^{*}\right)^{1 / 2}$ up to a sign. Comparing leading terms we find that $D L^{1 / 2} D^{-1}=\left(L^{*}\right)^{1 / 2}$. On the other hand, $\left(\left(L^{1 / 2}\right)^{*}\right)^{2}=L^{*}$, whence $\left(L^{1 / 2}\right)^{*}$ must be $\left(L^{*}\right)^{1 / 2}$ up to a sign. Comparing leading terms we find that we must chose the - sign, whence $\left(L^{n / 2}\right)^{*}=(-1)^{n}\left(L^{*}\right)^{n / 2}$. Thus, $\left(L^{n / 2}\right)^{*}=(-1)^{n} D L^{n / 2} D^{-1}$ as we had claimed.

We now write down the first two equations in the hierarchy. For this it is necessary to compute $L^{1 / 2}$ to sufficiently high (i.e. , negative) order. It shall be convenient to write $L^{1 / 2}$ up to order $D^{-9}$. 
Lemma 4.7. If $L=D^{4}+U D$, then its square root is given by $L^{1 / 2}=D^{2}+$ $\sum_{i \geq 1} a_{i} D^{-i}$, where the first nine coefficients are given by

and

$$
\begin{aligned}
& a_{1}=\frac{1}{2} U, \quad a_{2}=0, \quad a_{3}=-\frac{1}{4} U^{\prime \prime}, \quad a_{4}=0 \\
& a_{5}= \frac{1}{8}\left(U^{[4]}-U U^{\prime}\right), \quad a_{6}=-\frac{1}{8} U U^{\prime \prime}, \\
& a_{7}=\frac{1}{4} U U^{\prime \prime \prime}+\frac{1}{8} U^{\prime} U^{\prime \prime}-\frac{1}{16} U^{[6]}, \quad a_{8}=\frac{1}{4} U U^{[4]}
\end{aligned}
$$

$$
a_{9}=\frac{1}{32} U^{[8]}+\frac{1}{16} U\left(U^{\prime}\right)^{2}-\frac{11}{32} U U^{[5]}-\frac{3}{32} U^{\prime} U^{[4]}-\frac{11}{32} U^{\prime \prime} U^{\prime \prime \prime}
$$

From this it follows that $L_{+}^{1 / 2}=D^{2}$ and $L_{+}^{3 / 2}=D^{6}+\frac{3}{2} U D^{3}+\frac{3}{4} U^{\prime \prime} D$; whence the first two equations of the hierarchy are:

and

$$
\frac{\partial U}{\partial t_{1}}=U^{\prime \prime}
$$

$$
\frac{\partial U}{\partial t_{3}}=\frac{1}{4} U^{[6]}+\frac{3}{4}\left(U U^{\prime}\right)^{\prime \prime}
$$

which are, respectively, the supersymmetric chiral wave equation and the supersymmetric extension of the KdV equation found by Manin and Radul ${ }^{[4]}$ by the same Lax representation.

We now investigate the induced Poisson structures. Let $M$ denote the space of Lax operators of the form $D^{4}+\cdots$, and $M_{o}$ denote the submanifold of Lax operators $L$ obeying $L^{*}=D L D^{-1}$. As we will show, $M_{o}$ will inherit both Poisson structures from $M$. Strictly speaking, we cannot say that $M_{O}$ is a symplectic submanifold of $M$ with respect to both Poisson structures - not even in a formal sense - since the first Poisson structure is degenerate. Nevertheless, the second structure is (formally) nondegenerate and will allow us to define on $M_{o}$ a welldefined split of vector fields into those tangent to $M_{O}$ and those (symplectically) normal to $M_{o}$. This is important in order to define, for example, the gradient 
of a function on $M_{o}$ as a 1-form on $M$. An embedding of manifolds induces an embedding of their tangent bundles, but not of their cotangent bundles, since this is equivalent to a choice of normal bundle for the embedded submanifold. In the symplectic category there is a natural choice, since we then have a notion of symplectically normal vectors. Thus, a 1-form on $M$ defines a 1-form on $M_{O}$ if and only if the vector field associated to it via the symplectic form is tangent to $M_{o}$. This only makes sense, however, if the symplectic form is nondegenerate when restricted to $M_{o}$, which is precisely the condition that the embedded submanifold be symplectic. In our case we have two Poisson structures: one of which - the secondis (formally) nondegenerate; whereas the other - the first - is degenerate. Thus, using the second structure we can specify 1-forms on $M_{O}$ uniquely, whereas with the first structure some ambiguity remains. In principle it could happen that the ambiguity in one specification is incompatible with the other but here, remarkably, this is not the case. Hence both structures will turn out to be induced and, as a consequence, the bihamiltonian structure will be preserved under reduction.

Proposition 4.10. Let $X=\sum_{i=0}^{3} D^{-i-1} X_{i}$ be a 1 -form on $M$. Then, relative to the (second) Poisson structure $J_{0}$, it defines a 1 -form on $M_{o}$ if and only its coefficients satisfy the following conditions:

$$
X_{2}=-\frac{1}{2} X_{1}^{\prime}, \quad X_{3}=\frac{1}{2} X_{1}^{\prime \prime}, \text { and } X_{1}^{\prime \prime}=-\left(X_{0}^{\prime \prime \prime}+U X_{0}\right)
$$

Proof: Let $X=\sum_{i=0}^{3} D^{-i-1} X_{i}$ be a 1 -form on $M$. Relative to $J_{0}$ it defines a 1 -form on $M_{o}$ if and only if the vector field $J_{0}(X)=(L X)_{+} L-L(X L)_{+}$is tangent to $M_{o}$. In other words, writing $J_{0}(X)=\sum_{i=0}^{3} Y_{i} D^{i}$, we require that all the $Y_{i}$ are zero except for $Y_{1}$. A somewhat tedious computation yields 


$$
\begin{aligned}
& Y_{3}=(-1)^{|X|}\left[2 X_{2}^{\prime \prime}-X_{0}^{[4]}-\left(U X_{0}\right)^{\prime}\right], \\
& Y_{2}=X_{0}^{[5]}+\left(U X_{0}\right)^{\prime \prime}-2 X_{2}^{\prime \prime \prime}-2 X_{3}^{\prime \prime}-U X_{1}^{\prime}-2 U X_{2}+X_{1}^{[4]}, \\
& Y_{1}=(-1)^{|X|}\left[X_{2}^{[4]}-X_{0}^{[6]}-\left(U X_{0}\right)^{\prime \prime \prime}+2 U X_{1}^{\prime \prime}+U^{\prime \prime} X_{1}-\left(U X_{2}\right)^{\prime}\right],
\end{aligned}
$$

and

$$
\begin{aligned}
Y_{0}= & X_{0}^{[7]}+X_{1}^{[6]}-X_{2}^{[5]}-X_{3}^{[4]}+\left(U X_{0}\right)^{[4]}+U X_{0}^{[4]}+U X_{1}^{\prime \prime \prime} \\
& -U X_{2}^{\prime \prime}-U X_{3}^{\prime \prime}+U U^{\prime} X_{0} .
\end{aligned}
$$

Setting $Y_{3}=Y_{2}=Y_{0}=0$ we find the conditions listed above.

On the other hand, the first Poisson structure does not determine the coefficients of the one-form uniquely, but the constraints it imposes on a given 1-form are compatible with the ones imposed by the second Poisson structure.

Proposition 4.11. Let $X=\sum_{i=0}^{3} D^{-i-1} X_{i}$ be a 1-form on $M$. Then, relative to the (first) Poisson structure $J_{\infty}$, it defines a 1-form on $M_{o}$ as long as its coefficients satisfy

$$
X_{1}^{\prime \prime}=-\left(X_{0}^{\prime \prime \prime}+U X_{0}\right)
$$

Proof: This follows similar lines to that of the previous proof, except that now it is $J_{\infty}(X)=[L, X]_{+}$that must be tangent to $M_{o}$. Writing $J_{\infty}(X)=\sum_{i=0}^{3} Y_{i} D^{i}$, we find that $Y_{3}=Y_{2}=0, Y_{1}=-(-1)^{|X|} 2 X_{0}^{\prime \prime}$, and $Y_{0}=2 X_{1}^{\prime \prime}+2 X_{0}^{\prime \prime \prime}+2 U X_{0}$. Setting $Y_{0}=0$ we find the condition stated above.

We can now compute the induced Poisson structures. These can be computed by the Dirac bracket prescription or, equivalently, by using the Poisson brackets on $M$ but with the gradients defined in such a way that they define 1-forms on $M_{o}$. In other words, given two functions $F$ and $G$ defined on $M_{o}$ to compute their gradients we first extend them to functions on $M$, which we also denote by $F$ and $G$. Clearly, on $M_{o}, \frac{\delta F}{\delta U}$ and $\frac{\delta G}{\delta U}$ are unambiguously determined; but this is not the case for the variations with respect to the other coordinates since this depends on 
the extension. Nevertheless, Proposition 4.10 fixes them unambiguously in terms of $\frac{\delta F}{\delta U}$.

Theorem 4.12. The Poisson structures induced by $J_{\infty}$ and $J_{0}$ on $M_{o}$ are given by

$$
\{F, G\}_{\infty}=-\int_{B}\left(\widetilde{J}_{\infty} \cdot \frac{\delta F}{\delta U}\right) \frac{\delta G}{\delta U}
$$

and

$$
\{F, G\}_{0}=-\int_{B}\left(\widetilde{J}_{0} \cdot \frac{\delta F}{\delta U}\right) \frac{\delta G}{\delta U}
$$

where

$$
\widetilde{J}_{\infty}=2 D^{2}\left(D^{3}+U\right)^{-1} D^{2}
$$

and

$$
\widetilde{J}_{0}=\frac{1}{2} D^{5}+\frac{3}{2} U D^{2}+\frac{1}{2} U^{\prime} D+U^{\prime \prime} .
$$

Moreover, the two brackets are coordinated.

Proof: We first compute $J_{0}(d F)$ and $J_{\infty}(d F)$ and then use (3.23) and (3.24) . We write $d F=\sum_{i=0}^{3} D^{-i-1} X_{i}$ where $X_{1}=-\frac{\delta F}{\delta U}$ and the other coefficients are determined from this by the relations in Proposition 4.10 . From the calculation in the proof of Proposition 4.10 we find

$$
J_{0}(d F)=-\left[(-1)^{|F|}\left(\frac{1}{2} D^{5}+\frac{3}{2} U D^{2}+\frac{1}{2} U^{\prime} D+U^{\prime \prime}\right) \cdot \frac{\delta F}{\delta U}\right] D
$$

and from the proof of Proposition 4.11

$$
J_{\infty}(d F)=-\left[(-1)^{|F|} 2 D^{2}\left(D^{3}+U\right)^{-1} D^{2} \cdot \frac{\delta F}{\delta U}\right] D
$$

Plugging this into (3.23) and (3.24) yields the desired result. To see that they are coordinated it suffices to note that for any two functions on $M$ the two brackets are coordinated, and the expressions we have just obtained for the brackets on $M_{o}$ correspond to the bracket on $M$ evaluated in particular extensions of the functions from $M_{o}$ to $M$. 
Let us denote the restriction of the conserved charges $H_{n}=-\frac{2}{n} \operatorname{Str} L^{n / 2}$ to $M_{o}$ also by $H_{n}$. Of course, the charges for $n$ even are automatically trivial. The first three nontrivial charges can be easily computed from the results of Lemma 4.7 :

$$
\begin{aligned}
& H_{1}=-\int_{B} U, \\
& H_{3}=-\frac{1}{4} \int_{B} U U^{\prime},
\end{aligned}
$$

and

$$
H_{5}=\frac{1}{16} \int_{B}\left(U^{\prime \prime} U^{\prime \prime \prime}-2 U\left(U^{\prime}\right)^{2}\right)
$$

In fact, we will see that, for $n$ odd, the $H_{n}$ are all nontrivial. But to prove this we need to exhibit the relation between the $H_{n}$ and the Lax flows.

Proposition 4.20. The Lax flow $\frac{\partial L}{\partial t_{n}}=\left[L_{+}^{n / 2}, L\right]$ is hamiltonian and is generated by $H_{n+2}$ relative to the first hamiltonian structure and by $H_{n}$ relative to the second.

Proof: We can take $d H_{n}=L_{-}^{\frac{n}{2}-1} \bmod D^{-4}$ for $L$ in $M_{o}$ since, by Proposition $4.5, J_{\infty}\left(L_{-}^{n / 2}\right)=J_{0}\left(L_{-}^{\frac{n}{2}-1}\right)$ is tangent to $M_{o}$. The Lax equations are given by $\frac{\partial L}{\partial t_{n}}=J_{0}\left(d H_{n}\right)=J_{\infty}\left(d H_{n+2}\right)$ still after reduction; and since the gradients $d H_{p}$ of all the conserved charges obey the conditions of Proposition 4.10 , this translates into

$$
\begin{aligned}
\frac{\partial U}{\partial t_{n}} & =-\left(\frac{1}{2} D^{5}+\frac{3}{2} U D^{2}+\frac{1}{2} U^{\prime} D+U^{\prime \prime}\right) \cdot \frac{\delta H_{n}}{\delta U} \\
& =-2 D^{2}\left(D^{3}+U\right)^{-1} D^{2} \cdot \frac{\delta H_{n+2}}{\delta U}
\end{aligned}
$$

which proves what we claimed.

As an immediate corollary we find Lenard relations between these charges.

Corollary 4.21. (Lenard relations) For all $n \in \mathbb{N}$, the following relation hold

$$
2 D^{2}\left(D^{3}+U\right)^{-1} D^{2} \cdot \frac{\delta H_{n+2}}{\delta U}=\left[\frac{1}{2} D^{5}+\frac{3}{2} U D^{2}+\frac{1}{2} U^{\prime} D+U^{\prime \prime}\right] \cdot \frac{\delta H_{n}}{\delta U} .
$$


The Lenard relations allow us to give a different proof of the involutivity of the conserved charges.

Proposition 4.23. For all $m, n \in \mathbb{N}$,

$$
\left\{H_{m}, H_{n}\right\}_{\infty}=\left\{H_{m}, H_{n}\right\}_{0}=0 .
$$

Proof: The Lenard relations say that the Poisson flows generated by $H_{n}$ relative to the second Poisson structure and the one generated by $H_{n+2}$ relative to the first coincide. In other words, for all functions $H$,

$$
\left\{H, H_{n}\right\}_{2}=\left\{H, H_{n+2}\right\}_{1} \text {. }
$$

In particular, when $H$ is one of the conserved quantities, say $H_{m}$, one has that

$$
\begin{aligned}
\left\{H_{m}, H_{n}\right\}_{2} & =\left\{H_{m}, H_{n+2}\right\}_{1} \\
& =\left\{H_{m-2}, H_{n+2}\right\}_{2} \\
& \vdots \\
& =\left\{H_{m-2 j+2}, H_{n+2 j}\right\}_{1} \\
& =\left\{H_{m-2 j}, H_{n+2 j}\right\}_{2}
\end{aligned}
$$

Assuming, for definiteness, that $m>n$ we find in the above chain of equations either $\left\{H_{q}, H_{q}\right\}_{1}$ or $\left\{H_{q}, H_{q}\right\}_{2}$ for some $q$, both of which vanish since $\left|H_{q}\right|=0$ for all $q$. Therefore we find that the conserved charges are in involution with respect to both Poisson structures.

Finally it remains to prove that the conserved charges are nontrivial. We showed in the previous section ( $c f$. Corollary 3.34 ) that this was the case on $M$. However after setting $U_{0}=U_{2}=U_{3}=0$ it would not be inconceivable that some of them do not survive the reduction. Still, one has the following. 
Proposition 4.26. For all $n$ odd, the conserved charges $H_{n}$ are nontrivial.

Proof: Let $n$ be odd and suppose that $H_{n+2}=0$. Then by Proposition $4.20 \frac{\partial L}{\partial t_{n}}=$ 0 , which forces $L_{+}^{n / 2}$ to belong to $Z_{L}$. By the usual grading arguments it follows that $L_{+}^{n / 2}=L^{n / 2}$, whence $H_{n}=0$. Eventually we reach at $H_{1}=0$, which is absurd as evidenced by (4.17) .

This concludes the proof of the hamiltonian integrability of the SKdV hierarchy.

\section{$\S 5$ Conclusions}

In this paper we have studied a class of supersymmetric integrable systems which arise as reductions of the even order SKP hierarchy. The resulting hierarchies consists of nonlinear evolution equations given in Lax form. We have shown that these equations are bihamiltonian with respect to the supersymmetric analogs of the Gel'fand-Dickey brackets constructed in [6] . The hamiltonian functions generating the flows are given by the supertrace of fractional powers of the relevant Lax operator. They are shown to satisfy (Lenard) recursion relations and to be in involution relative to both Poisson brackets and, hence, relative to any linear combination of them - since they are coordinated.

The evolution equations for the resulting hierarchies - even the simplest one are rather complicated; although — as shown in section 4-by further reductions one finds more tractable systems. In particular, a reduction of the simplest hierarchy is identified with the supersymmetric extension of the KdV equation ( $\mathrm{SKdV}$ ) found by Manin and Radul ${ }^{[4]}$. Moreover all properties pertaining to the bihamiltonian nature of the hierarchy are induced under reduction and this has allowed us to discover the missing "first" hamiltonian structure of the SKdV equation and to prove the hamiltonian integrability of the hierarchy.

This fact suggests that interesting supersymmetric hierarchies can be ontained by reduction of the higher order SKdV hierarchies treated here. This is an interesting open problem to which we hope to turn our attention in a future publication. 
Another interesting by-product of these reductions is the appearance of extended superconformal algebras ( $W$-superalgebras) as the fundamental Poisson brackets relative to the second hamiltonian structure. Already before reduction one can show that the fundamental Poisson brackets relative to the second supersymmetric Gel'fand-Dickey bracket contain a superconformal algebra. Indeed, the two superfields of weights 1 and $\frac{3}{2}$ can be suitably redefined (by addition of derivatives of superfields of lower weight) in such a way that the resulting superfield of weight $\frac{3}{2}$ generates the $N=1$ supervirasoro algebra and such that the one of weight 1 is a classical superconformal tensor of conformal weight 1 . In [9] and [12] $W$-superalgebras were constructed by reduction of the second supersymmetric Gel'fand-Dickey bracket for odd order Lax operators. It is an interesting question to ask what reductions can be performed on even order Lax operators to obtain $W$-superalgebras.

Reductions are also interesting from the viewpoint of string theory and statistical mechanics, where $W$-superalgebras and, in particular, their unitary highest weight representations as operators in a two-dimensional superconformal quantum field theory, play a very important role. The analysis of these representations usually proceeds by first embedding the relevant $W$-superalgebra inside a free field algebra as classical algebras. Then this embedding is quantized to obtain a quantum free field realization. For $W$-(super)algebras related to integrable systems the classical embedding is given by the Miura transformation. Now, as shown in [6] , for the $W$-superalgebras obtained from supersymmetric Lax operators, the natural (Fock) representations of the free field algebra are not unitary, since the free fields have alternating signs in their Poisson brackets - equivalently, in their (anti)commutation relations. One way to avoid this problem-and thus to obtain manifestly unitary representations without further ado - would be to find a reduction which, at the level of the free fields, leaves only those fields with the same sign in their Poisson bracket. Particularly trivial examples of this are the $N=1$ 
and $N=2$ supervirasoro algebras obtained as the simplest examples of the $W$ superalgebras of [9] and [12], respectively. The search for further reductions of this type is in progress.

\section{ACKNOWLEDGEMENTS}

We extend our thanks and appreciation to the Instituut voor Theoretische Fysica of the Universiteit Leuven and to the Departamento de Física de Partículas Elementales of the Universidad de Santiago de Compostela for their hospitality and financial support throughout this collaboration.

\section{REFERENCES}

[1] E. Date, M. Jimbo, M. Kashiwara, and T. Miwa, Proc. Jpn. Acad. Sci. 57A (1981) 387; J. Phys. Soc. Jpn. 50 (1981) 3866.

[2] V. G. Drinfel'd and V. V. Sokolov, J. Soviet Math. 30 (1984) 1975.

[3] L. A. Dickey, Integrable equations and Hamiltonian Systems, World Scientific Publishing Co. in press.

[4] Yu. I. Manin and A. O. Radul, Comm. Math. Phys. 98 (1985) 65.

[5] I. M. Gel'fand and L. A. Dickey, A family of Hamiltonian structures connected with integrable nonlinear differential equations, Preprint 136, IPM AN SSSR, Moscow (1978).

[6] J.M. Figueroa-O'Farrill and E. Ramos, Phys. Lett. 262B (1991) 265.

[7] J.M. Figueroa-O'Farrill, J. Mas, and E. Ramos, Bihamiltonian Structure of the Supersymmetric SKdV Hierarchy, Leuven Preprint KUL-TF-91/19 (April 1991).

[8] Y. Watanabe, Letters in Math. Phys. 14 (1987) 263.

[9] J.M. Figueroa-O'Farrill and E. Ramos, Classical $W$-superalgebras from hamiltonian reduction, Preprint-KUL-TF-91/14 (March 1991). 
[10] B.A. Kupershmidt and G. Wilson, Invent. math. 62 (1981) 403.

[11] L. A. Dickey, Comm. Math. Phys. 87 (1982) 127.

[12] J.M. Figueroa-O'Farrill and E. Ramos, Supersymmetric Gel'fand-Dickey brackets and classical $N=2 W$-superalgebras, Preprint-KUL-TF-91/13 (March 1991). 